\title{
Dez anos de cirurgia dos aneurismas e dissecções crônicas da aorta ascendente no Instituto do Coração - FMUSP
}

\author{
Ronaldo Ducceschi FONTES*, Noedir A. G. STOLF*, Domingos D. LOURENÇO FILHO*, Ricardo TRAN-
} $\mathrm{CHESI}^{\star}$, Charles MADY*, Antônio Carlos PEREIRA-BARRETO*, Fúlvio PILEGGI*, Adib D. JATENE*

\section{RBCCV 44205-128}

FONTES, R. D.; STOLF, N. A. G.; LOURENÇO FILHO, D. D.; TRANCHESI, R.; MADY, C.; PEREIRABARRETO, A. C.; PILEGGI, F.; JATENE, A. D. - Dez anos de cirurgia dos aneurismas e dissecçōes crônicas da aorta ascendente no Instituto do Coraçāo - FMUSP. Rev. Bras. Cir. Cardiovasc., 6(1): 24-29, 1991.

RESUMO: Entre janeiro de 1980 e dezembro de 1990, 109 pacientes com idade entre 12 e 70 anos, 86 do sexo masculino e 23 do sexo feminino, foram operados para tratamento de aneurismas e dissecçōes da aorta ascendente, associados ou nāo a insuficiência aórtica. Trinta e quatro pacientes estavam em classe funcional (CF) IV (NYHA), 51 em CF III, 18 em CF II e seis em CF I. Cinqüenta e dois pacientes tinham dissecção crônica da aorta, 29 tinham ectasia ânulo-aórtica, dez aneurisma sacular com insuficiência aórtica e os demais, diagnósticos associados. A mortalidade imediata foi de $12,8 \%$ (14 óbitos). Vinte e sete $(24,7 \%)$ pacientes não foram acompanhados tardiamente. A mortalidade tardia foi de $13,4 \%(11 / 82)$. Dos 72 pacientes acompanhados clinicamente até 120 meses de evolução (três a 120 meses), 65 (90,5\%) mantêm-se em CF I e II. Concluiu-se que: a operação de Bentall - De Bono, demonstra ter melhor resultado em relaçăo às interposiçōes de tubo $(p<0,01)$, com estimativa de funçăo de sobrevida de $70 \%$ em 120 meses, com excelente evolução clínica tardia.

DESCRITORES: aneurismas de aorta ascendente, cirurgia; dissecçōes de aorta ascendente, cirurgia.

\section{INTRODUÇĀO}

A reconstruçāo da aorta ascendente é necessária em várias doenças, especialmente nas lesōes distróficas da aorta, necrose cística, idiopática, sífilis e aterosclerose ${ }^{1}$.

As lesões mais comuns e que freqüentemente necessitam intervençāo sobre a aorta são os aneurismas e as dissecçōes da aorta ascendente associados ou nāo a insuficiêneia valvar ${ }^{7}$.

Os aneurismas são encontrados em duas situações básicas: envolvendo a raiz da aorta e os seios de Val- salva (ectasia ânulo-aórtica) ou somente parte da aorta ascendente acima dos óstios coronários (aneurisma supracoronário) ${ }^{7}$.

A intervenção sobre a aorta ascendente é recomendada para pacientes portadores de complicaçōes que incluem obstrução de ramos arteriais, dor torácica persistente, dilatação aórtica acima de $5,5 \mathrm{~cm}$, ou que tenham sinais de rotura iminente ${ }^{9}$.

Várias técnicas têm sido utilizadas para tratamento cirúrgico desses aneurismas e, associadas aos avanços tecnológicos alcançados nas últimas décadas, têm contribuído para melhora dos resultados operatórios ${ }^{10}$

Trabalho realizado no Instituto do Coração do Hospital das Clínicas da Faculdade de Medicina da Universidade de São Paulo. Săo Paulo, SP, Brasil.

Apresentado ao 18: Congresso Nacional de Cirurgia Cardiaca. Rio de Janeiro, RJ, 5 e 6 de abril, 1991

- Do Instituto do Coraçáo do Hospital das Clínicas da Faculdade de Medicina da Universidade de Săo Paulo.

Endereço para sepe a!ıs: Ronaldo D. Fontes. Av. Dr. Enéas de Carvalho Aguiar, 44. Divisāo Cirúrgica. 05403 São Paulo, SP, Brasil 
FONTES, R. D.; STOLF, N. A. G.; LOURENÇO FILHO, D. D.; TRANCHESI, R.; MADY, C.; PEREIRA-BARRETO, A. C.; PILEGGI, F.; JATENE, A. D. - Dez anos de cirurgia dos aneurismas e dissecçōes crônicas da aorta ascendente no Instituto do Coração - FMUSP. Rev. Bras. Cir. Cardiovasc., 6(1): 24-29, 1991.

Por esta razăo, estamos revisando os resultados imediatos e tardios de nossa série de pacientes, que compreende os últimos dez anos.

\section{CASUÍSTICA E MÉTODOS}

Entre janeiro de 1980 e dezembro de 1990, 109 pacientes foram operados no Instituto do Coração FMUSP, para tratamento de aneurismas e dissecções crônicas da aorta ascendente.

Oitenta e seis pacientes eram do sexo masculino e 23 do sexo feminino, com média de idade de 53,6 anos, variando entre 12 e 70 anos. A maioria dos pacientes apresentava dilatação da aorta ascendente com severa insuficiência valvar aórtica.

As duas indicaçōes mais freqüentes para o tratamento cirúrgico foram: insuficiência cardiaca determinada pela insuficiência aórtica e dor torácica relacionada com a expansão do aneurisma.

Trinta e quatro pacientes estavam em classe funcional (CF) IV (NYHA) 51 em CF III, 18 em CF II, e seis em CF I. Os pacientes que estavam em classe funcional I e II (24 pacientes), foram operados em razāo da expansão do aneurisma e da dor torácica.

Conqüena e dois pacientes tinham diagnóstico de dissecçăo crônica de aorta, 29 de ectasia ânulo-aórtica, dez de aneurisma sacular com insuficiência valvar aórtica, quatro de aneurisma sacular sem insuficiência valvar aórtica, três de dissecção crônica de aorta com insuficiência aórtica e mitral, dois de aneurisma sacular com insuficiência aórtica e mitral, dois de aneurisma sacular com disfunçăo de prótese aórtica, três de aneurisma sacular com insuficiência aórtica e coronária, dois de ectasia ânulo-aórtica com insuficiência coronária, um aneurisma de aorta ascendente com disfunçāo de prótese aórtica e insuficiência mitral e o último, de aneurisma de aorta ascendente com calcificação total da aorta com insuficiência aórtica e coronária, perfazendo um total de 109 pacientes.

\section{RESULTADOS}

A mortalidade total imediata foi de $12,8 \%$ (14 pacientes), cinco deles por baixo débito cardiaco, seis por sangramento (dois através da malha do tubo, um na sutura da coronária direita e três por coagulopatia dilucional), um por síndrome da angústia respiratória do adulto, um por infarto agudo do miocárdio e um por acidente vascular cerebral.

No período compreendido entre 1980 e 1983, 20 pacientes foram operados, dos quais cinco $(25 \%)$ falece- ram; emtre 1984 e 1987, foram operados 41 pacientes, com três $(7,3 \%)$ óbitos e, entre 1988 e 1990,48 pacientes, com seis $(12,5 \%)$ obitos.

Apesar da diferença entre os resultados nos períodos distintos, esta năo foi estatisticamente significante (Figura 1).

$\mathrm{Na}$ análise da mortalidade por diagnóstico pré-operatório, também não houve diferença significativa; entretanto, é de notar que 25 pacientes eram portadores de ectasia ânulo-aórtica e quatro (16\%) deles faleceram, 56 tinham dissecçăo crônica de aorta com insuficiência aórtica e cinco $(8,9 \%)$ pacientes faleceram no pós-operatóriọ imediato.

Dóze pacientes reoperados por sangramento no período pós-operatório imediato, seis dós quais foram a óbito (três por sangramento ativo e três por coagulopatia dilucional). Três dos 12 pacientes foram submetidos ao uso de compressas para o controle do sangramento intra-operatório, obtendo-se sucesso em dois.

A operação de Bentall e De Bono (procedimento A), foi realizada em 46 pacientes, dos quais $(4,3 \%)$ faleceram; interposiçăo de tubo de Dacrón sem substituição valvar (procedimento B) foi realizado em 33 pacientes, com cinco $(15,1 \%)$ óbitos; interposição de tubo de Dacron com substituição valvar aórtica (procedimento C) foi utilizada para 18 pacientes, dos quais cinco $(27,7 \%)$ faleceram. A análise estatística através do Qui-quadrado ${ }^{18}$ confronta os resultados dos procedimentos em relação à mortalidade e demonstra que a operação de Bentall tem resultado superior aos das interposiçōes de tubo $(p<0,01)$ (Figura 2).

Quando se divide as interposiçōes de tubo de Dacron com substituição vaivair e sem substituição valvar, comparanco-as separadamente com o procedimento de Bentall, verifica-se que o resultado da operaçăo de Bentall é superior ao da interposiçăo com substituição valvar ( $p<0,01)$ e năo é significativarnente diferente quando comparado com as interposiçōes sem substituição valvar.

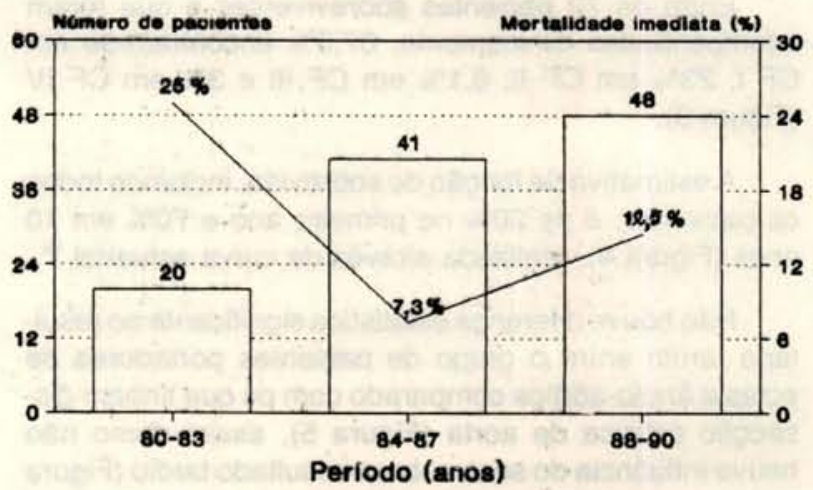

Fig. 1 - Distribuiçăo por periodos e respectiva mortalidade. 
FONTES, R. D.; STOLF, N. A. G.; LOURENÇO FILHO, D. D.; TRANCHESI, R.; MADY, C.; PEREIRA-BARRETO, A. C.; PILEGGI,

F.; JATENE, A. D. - Dez anos de cirurgia dos aneurismas e dissecçōes crônicas da aorta ascendente no Instituto do Coração - FMUSP. Rev. Bras. Cir. Cardiovasc., 6(1): 24-29, 1991.

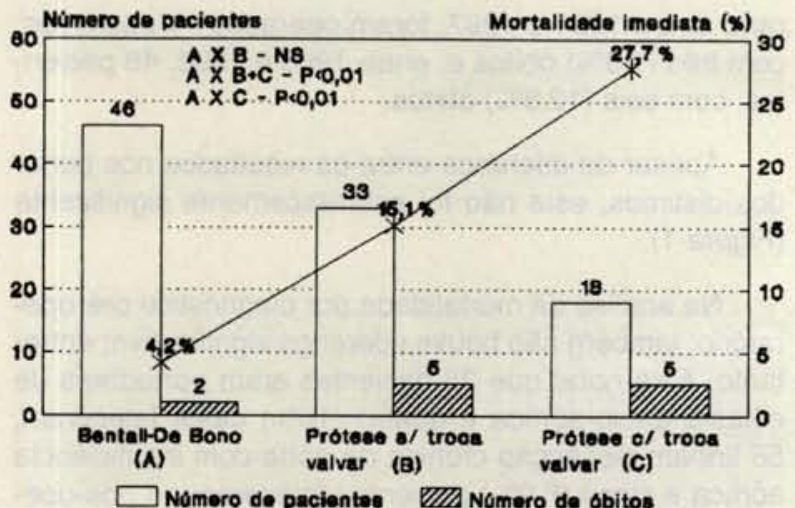

Fig. 2-Resultado dos diferentes procedimentos.

Procedimentos associados foram realizados em 12 pacientes, com mortalidade imediata e $25 \%$ (três óbitos). Correção do aneurisma com substituição valvar e revascularização do miocárdio foi realizada em dois pacientes, com um óbito, correçāo do aneurisma e revascularização do miocárdio em cinco pacientes, com um óbito, operação de Bentall com substituição valvar mitral e revascularizaçăo do miocárdio em um paciente, operação de Bentall com revascularização do miocárdio em três pacientes, com um óbito, e o último foi submetido a correção do aneurisma com substituição valvar aórtica e aneurisma de parte membranácea do septo.

\section{Evolução Tardia}

Foram seguidos clinicamente, ou obtidas informações por comunicação pessoal, $83(76,1 \%)$ pacientes, dos quais $11(13,2 \%)$ faleceram, quatro por morte súbita, dois por insuficiência cardiaca em 90 e 120 meses de evolução, dois por infarto do miocárdio em quatro e seis meses de acompanhamento, um por hemorragia e tamponamento cardiaco tardio aos 50 dias de pós-operatório, outro por expansāo e rotura do aneurisma em outra localização e um por acidente vascular cerebral após 36 meses de seguimento.

Entre os 72 pacientes sobreviventes e que foram acompanhados clinicamente, $67,5 \%$ encontram-se em CF I, $23 \%$ em CF II, $6,1 \%$ em CF III e $3 \%$ em CF IV (Figura 3).

A estimativa de função de sobrevida, incluindo todos os pacientes, é de $20 \%$ no primeiro ano e $70 \%$ em 10 anos (Figura 4), realizada através de curva actuarial ${ }^{18}$.

Não houve diferença estatística significante ao resultado tardio entre o grupo de pacientes portadores de ectasia ânulo-aórtica comparado com os que tinham dissecção crônica de aorta (Figura 5), assim como nāo houve influência do sexo sobre o resultado tardio (Figura $6)$.

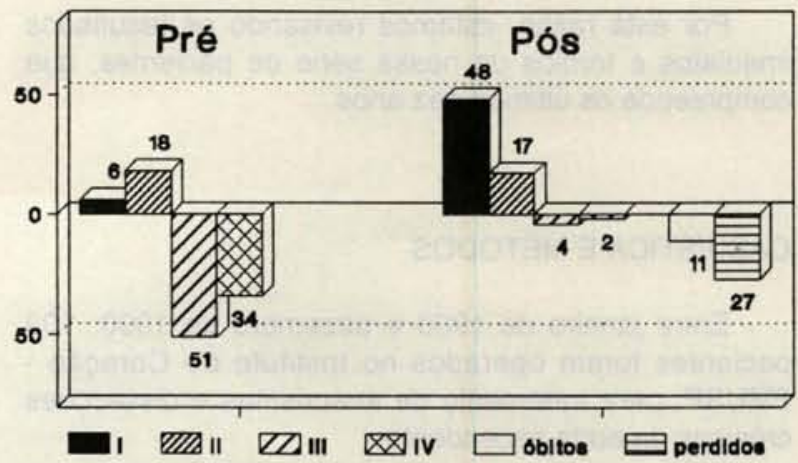

Fig. 3-Classe funcional. Significativa melhora do estado clinico pósoperatório.

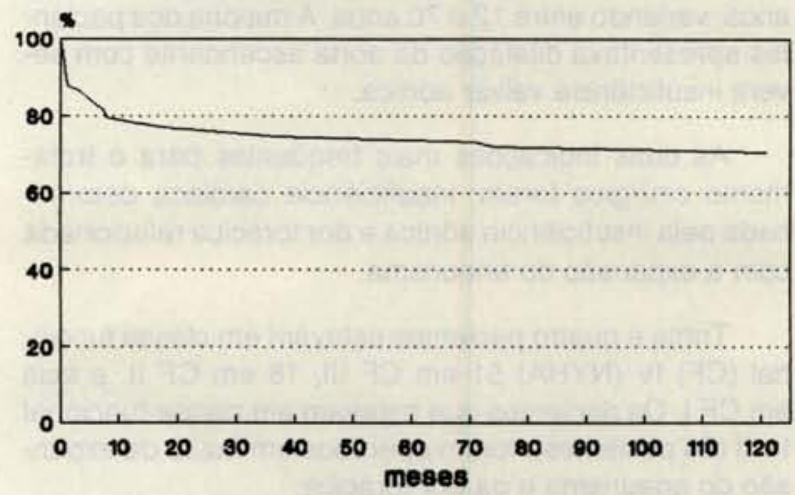

Fig. 4 - Curva atuanal 1980-1990. Expectativa de sobrevida para todos os pacientes em 120 meses.

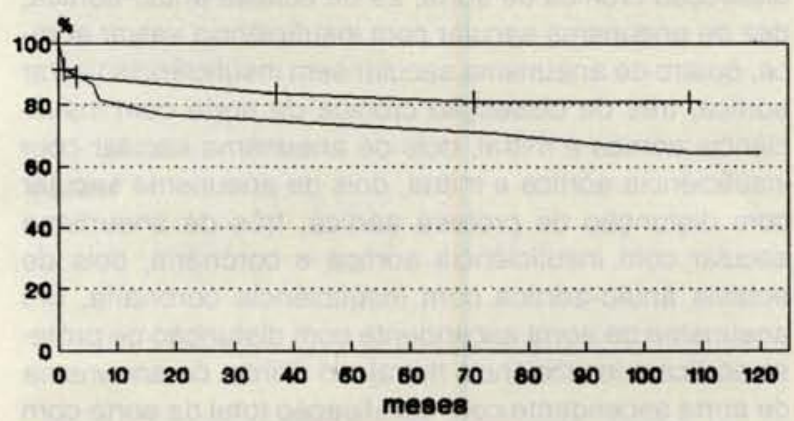

Fig. 5-Curva atuarial 1980-1990. Diagnóstico. Expectativa de vida em 120 meses em portadores de ectasia ou aneurisma de aorta ascencente.

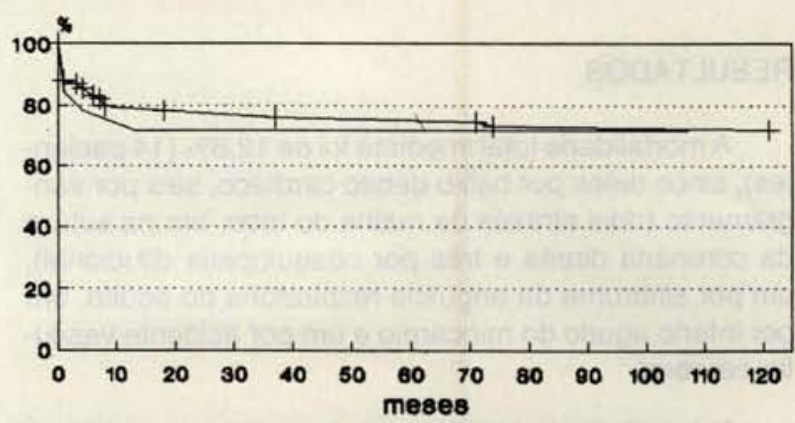

Fig. 6-Curva atuaria 1980-1990. Expectativa de vida em 120 meses quanto ao sexo. 
FONTES, R. D.; STOLF, N. A. G.; LOURENÇO FILHO, D. D.; TRANCHESI, R.; MADY, C.; PEREIRA-BARRETO, A. C.; PILEGGI, F.; JATENE, A. D. - Dez anos de cirurgia dos aneurismas e dissecçōes crônicas da aorta ascendente no Instituto do Coraçâo - FMUSP. Rev. Bras. Cir. Cardiovasc., 6(1): 24-29, 1991.

\section{DISCUSSĀO}

O tratamento cirúrgico dos aneurismas e dissecções crônicas da aorta ascendente, associados ou não a disfunção valvar aórtica, tem apresentado inúmeros problemas técnicos que, com o decorrer dos anos, foram corrigidos através de diferentes procedimentos ${ }^{4,8}, 19$.

As técnicas inicialmente utilizadas, incluindo as ressecçōes parciais ou segmentares da aorta ascendente seguidas de anastomose direta, tinham alta mortalidade pela fragilidade da parede aórtica resultando em rotura ou dissecção da aorta remanescente ${ }^{12,19}$.

A descrição realizada por BENTALL \& De BONO ${ }^{4}$, em 1968, utilizando tubo valvulado e reimplantando os óstios coronários, demonstrou ser a solução racional do problema.

Entretanto, mesmo essa técnica não elimina complicações, tais como tração da parede aórtica sobre as anastomoses dos óstios coronários, hemorragia incontrolável determinada pelo difícil acesso ${ }^{2}$, levando a ocorrência tardia de formação de falso aneurisma na zona de reimplante das coronárias ${ }^{13}$, assim como dissecção da aorta remanescente.

Essas complicações foram amenizadas sem dúvida pelo aparecimento da cola biológica de Bachet (BACHET et alii $\left.{ }^{3}\right)$, CABROL et alii ${ }^{8}$ descreveram sua técnica para correçāo dos aneurismas de aorta ascendente, reestabelecendo a circulação coronária utilizando tubo de Dacron com oito a $10 \mathrm{~mm}$ de diâmetro, ao invés da anastomose direta do tubo valvulado com os óstios coronários e conseguiu, com isso, diminuir o sangramento incontrolável.

Outras contribuiçóes importantes foram conseguidas, através de avanços tecnológicos, na fabricação de próteses de Dacron, que se tornaram macias, mantiveram a baixa porosidade, facilitando a técnida operatória e diminuindo os problemas de pré-coagulação dos tubos. O aparecimento dos tubos compostos com próteses valvulares, produzidas industrialmente, eliminaram o problema das inserçōes obliquas das próteses que acarretavam a disfunção das válvulas artificiais ${ }^{10}$.

O desenvolvimento de hematoma ao redor das próteses tubulares, provocado por alteração de coagulação sangüinea, levando a compressấo e deslocamento da zona de anastomose dos óstios coronários, foi minimizado com a criação de fístula entre o espaço periprotético e o átrio direito, utilizando tubo de Gore-tex, até o restabelecimento total da coagulação do sangue ${ }^{6,8}$.

Portanto, essas complicaçōes mais importantes, que ocorrem no tratamento cirúrgico dos aneurismas de aorta ascendente, têm, realmente, recebido atenção constante dos diversos cirurgióes que se dedicam a esse tipo de tratamento ${ }^{1}, 11,14,15,17,20$.

Entretanto, sabe-se que a causa mais freqüente de morte tardia em pacientes operados de aneurisma de aorta é a formação de aneurisma em outra localização, com a conseqüente rotura ${ }^{9}$.

Particularmente para os procedimentos de Bentall e De Bono, ou de Wheat, a complicação tardia mais freqüente é a dissecção da aorta remanescente ${ }^{13,14}$ 16 .

Para diminuir essa complicação, pode-se utilizar a cola biológica de Bachet na sutura distal entre o tubo de Dacron e a aorta ${ }^{3}$, que confere maior aderência entre as lâminas e, conseqüentemente, mais firmeza nas suturas.

Em nosso meio, podemos inferir que, invariavelmente, os pacientes são encaminhados para tratamento cirúrgico em fase adiantada da doença, não só da valvopatia em si, como também da dilatação da aorta ascendente que, geralmente, excede em muito o tamanho admitido como limite mínimo para indicação cirúrgica.

Colocando-se à disposição da população em geral, a tomografia computadorizada, com o desenvolvimento das técnicas de arteriografia digital ou seriada, e a ressonância magnética, espera-se poder tratar esses pacientes em fase mais precoce da doença, diminuindo, com isso, a mortalidade imediata.

\section{CONCLUSÕES}

Os resultados obsevados, em termos de sobrevida e de melhora clínica, sugerem que a cirurgia é o tratamento de escolha para aneurismas e dissecçôes crônicas da aorta ascendente. 
FONTES, R. D.; STOLF, N. A. G.; LOURENÇO FILHO, D. D.; TRANCHESI, R.; MADY, C.; PEREIRA-BARRETO, A. C.; PILEGGI, F.; JATENE, A. D. - Dez ano6 de cirurgia dos aneurismas e dissecções crónicas da aorta ascendente no Instituto do Coraçăo - FMUSP. Rev. Bras. Cir. Cardiovasc., 6(1): 24-29, 1991.

RBCCV 44205-128

FONTES, R. D.; STOLF, N. A. G.; LOURENÇO FILHO, D. D.; TRANCHESI, R.; MADY, C.; PEREIRABARRETO, A. C.; PILEGGI, F.; JATENE, A. D. - Ten years of operation for aneurysms or chronic dissections of ascending aorta, in the Instituto do Coraçāo - FMUSP. Rev. Bras. Cir. Cardiovasc. 6(1): 24-29, 1991.

ABSTRACT: From January 1980 to Dezember 1990, 109 patients, 86 males and 16 females, ranging in age from 12 to 70 years, were operatated on for aneurysms or chronic dissections of the ascending aorta, associated or not to aortic valve insufficiency. Thirty-four patients were in New York Heart Association class IV, 51 in class III, 18 in class II and six in class I. Fifty-two patients had chronic aortic dissection, 29 annulo-aortic ectasia, 10 sacular aneurysm, remaining 8 ethiologics. The early mortality was $12.8 \%$ (14 deaths). Twenthy-seven patients were lost for follow-up during a period ranging from three months to 10 years (average 82 months). The late mortality was $13.4 \%(11 / 82)$. Among the 72 patients survivors, clinical improvement was observed in the majority of patients ( $90.5 \%$ are in class I or II). Among the several operative techniques, the Bentall and De Bono showed better early survival and is preferable option when indicated. The actuarial curve showed a $70 \%$ survival for the whole group, after 120 months. The results observed in terms of survival and clinical improvement suggest that surgery is the treatment of choice for aneurysms and chronic dissections of ascending aorta.

DESCRIPTORS: aneurysms of ascending aorta, surgery; dissections of ascending aorta, surgery.

\section{REFERÊNCIAS BIBLIOGRÁFICAS}

1 ACAR, J.; GUIMARD, A.; BANDOUY, P. A. - Les insuffisances aortiques par aneurysme dystrophique de l'aorte ascendante. Arch. Mal. Coeur., 72:596-605, 1979.

2 ASANO, K.; ANDO, T.; HANADA, S.; MARUYAMA, Y. Control of bleeding during the Bentall operation. $J$. Cardiovasc. Surg., 24: 13-14, 1983.

3 BACHET, J.; GIGOU, F.; LAURIAN, E.; BICAL, O.; GOUDOT, B.; DUBOIS, C.; BRODARY, D.; GULMET, D. - Quatre ans d'expérience clinique de la colle gelatine - resorcine - formol dans les dissections aigües de l'aorta ascendante. Arch. Mal. Coeur., 76: 87-94, 1983.

4 BENTALL, M. \& De BONO, A. - A techinique for complete replacement of ascending aorta. Thorax, 23: $388-390,1980$.

5 CABROL, C. - Annuloplastie valvulaire: un nouveau procédé. Presse Med., 1: 366-369, 1972.

6 CABROL, C.; GRANDHBAKHCH, I.; CHAM, B. - Aneurysmes de l'aorte ascendante: replacement total avec reimplantation des arteries coronaines. Presse Med., 7: 363-365, 1978.

7 CABROL, C.; GANDJBAKHCH, I.; PAVIE, A. - Surgical treatment of ascending aortic pathology. J. Cardiac. Surg., 3: 167-180, 1988.

8 CABROL, C.; PAVIE, A.; GRANBJDAKHCH, I. - Complete replacement of ascending aorta with reimplantation of the coronary arteries: new surgical approach. J. Thorac. Cardiovasc. Surg., 81: 309-315, 1981.

9 CRAWFORD, E. S. - Aortic dissection, Ann. Surg., 208: $251-273,1988$
10 CRAWFORD, E. S. - Progress in the treatment of thoracic aortic aneurysms. World J. Surg., 12: 805-809, 1988.

11 EDWARDS, W. S. \& KERR, A. A. - A safer technique for replacement of the entire ascending aorta and aortic valve. J. Thorac. Cardiovasc. Surg., 59: 837-839, 1970.

12 EGLOFF, L.; ROTHLIN, M.; KUGELMEIER, J.; SENNING, A.; TURINA, M. - The ascending aortic aneurysm: replace o repair? Ann. Thorac. Surg., 34: 117-124, 1982.

13 HASHIMOTO, A.; SHIMAZU, K.; NAKAMURA, K. - Later complication after composite replacement of anulo aortic ectasia. J. Cardiovasc. Surg., 24: 277-279, 1983.

14 HELSETH, H. K.; HAGLIN, J. J.; MANSON, B. K.; WICKSTRON, P. - Results of composite graft, replacement of aortic root aneurysms. J. Thorac. Cardiovasc. Surg., 80: 754-759, 1980.

15 MAYER, J. E.; LINDSAY, W. G.; WANG, Y.; JORGENSEN, C. R.; NICOLOFF, D. M. - Composite replacement of the aortic valve and ascending aorta. J. Thorac. Cardiovasc. Surg., 79: 816-824, 1978.

16 MILLER, D. C.; STINSON, E. B.; AFER, P. E.; MORENOCABRAL, R. I.; REITZ, B. A.; ROSSITER, S. J.; SHUMWAY, N. E. - Concomitant resection of the aortic valve: operative and long-term results with "conventional" techniques in ninety patients. J. Thorac. Cardiovasc. Surg., 79: 388-401, 1980.

17 ORSZULAK, T. A.; CLEMENTS, I. P.; TINKER, J. H. Björk-Shiley leaflet impairment in an ascending aortic conduit due to extrinsic compression by a false aneu. rysm. Ann. Thorac. Surg., 34: 706-709, 1982.

18 SIEGEL, S. - Estatística não paramétrica. São Paulo, Mc Graw-Hill, 1981, 350 p. 
FONTES, R. D.; STOLF, N. A. G.; LOURENÇO FILHO, D. D.; TRANCHESI, R.; MADY, C.; PEREIRA-BARRETO, A. C.; PILEGGI, F.; JATENE, A. D. - Dez anos de cirurgia dos aneurismas e dissecçōes crônicas da aorta ascendente no Instituto do Coração - FMUSP. Rev. Bras. Cir. Cardiovasc., 6(1): 24-29, 1991.

THEVENET, A. \& MARY, H. - Problèmes chirurgicaux particuliers des dissections du syndrome de Marfan. Le remplacement de l'aorte ascendante et de la valve aortique avec reimplantation de coronaires: a propos de 3 cas. Ann. Chir. Thorac. Cardiovasc., 33:632-636, 1979.

20 WHEAT Jr., M. N.; WILSON, J. R.; BARTLEY, T. D. Successful replacement of the entire ascending aorta and aortic valve. JAMA, 188: 717-719, 1964.

\section{Discussão
DR. PAULO P. PAULISTA
São Paulo, SP}

Os meus cumprimentos aos autores, pela apresentação do excelente trabalho, e as minhas congratulaçōes pelos dez anos festejados. Inicialmente, gostaria de esclarecer que nós, particularmente, não associamos os aneurismas verdadeiros, em geral decorrentes de aterosclerose ou lues, ainda presente em nosso meio, com os falsos aneurismas, conseqüentes do processo de dissecção aórtica, já que ambos têm etiologia, evolução e prognóstico diferentes. Em todo caso, com a finalidade de mostrar material semelhante, fizemos a associação dos aneurismas verdadeiros com os dissecantes crônicos, com cirurgia apenas na aorta ascendente, e encontramos, entre 1967 e 1991, um total de 263 pacientes, com 37 óbitos no hospital e $14,0 \%$ de mortalidade. Aumentamos o número total para 293, com 51 óbitos e $17,4 \%$ de mortalidade, ao incluirmos, em "dissecantes", todos os crônicos e também os agudos, pois estes originam aqueles. Houve acentuada diferença na mortalidade, muito menor nos aneurismas com associação de outros procedimentos, onde a mortalidade foi de $11,0 \%$, em contrapartida com as correçōes isoladas, onde ela foi de $39,3 \%$. A associação de implante de prótese ou de cirurgias de maior porte, como a de Bentall e De Bono, deram os índices mais baixos, com 6,2 e $7,1 \%$. Já a associação de revascularização do miocárdio foi fator de sensivel aumento na mortalidade, com $37,0 \%$.
Finalmente, desejaríamos lembrar que estamos com uma tendência em empregar a operação de Cabrol, em substituição à de Bentall e De Bono, já que a possibilidade de complicações decorrentes do reimplante dos óstios coronários na prótese da aorta ascendente ficam minimizados. Temos cinco casos, nos últimos seis meses, sem nenhum óbito. Novamente, cumprimentamos os autores do trabalho que comentamos e agradecemos o privilégio que a Comissão Organizadora nos deu.

\section{DR. FONTES \\ (Encerrando)}

Nós agradecemos ao Dr. Paulo Paulista os comentários sobre nosso trabalho, uma vez que ele tem contribuido para o tratamento de aneurismas de aorta em nosso meio, há vários anos. Em primeiro lugar, gostaria de esclarcer que as dissecçōes crônicas e os aneurismas da aorta ascendente foram agrupados na mesma casuística porque a evolução tardia do paciente operado, em nosso meio, foi estatisticamente semelhante. A revascularização miocárdica associada ao tratamento cirúrgico de aneurismas e dissecções de aorta ascendente teve um aumento não significativo na mortalidade e, nos últimos tempos, a freqüência de associação das duas doenças tem aumentado. Utilizamos a operação de Cabrol, conservando a valva aórtica, em dois pacientes, motivo de uma apresentação neste congresso; os dois pacientes evoluíram bem, sem insuficiência aórtica, porém um deles teve sangramento abundante durante $o$ ato operatório. Não acredito que a operação de Cabrol minimize as complicaçōes referentes ao reimplante das coronárias, pois os óstios coronários sāo inseridos na prótese tubular, sem possibilidade de revestir o enxerto com a "capa" do aneurisma, nem utilizar a "manobra" de Cabrol. Tenho algumas dúvidas ainda, quanto à normalidade do tecido valvar aórtico na doença ânulo-aórtica, a ponto de podermos preservá-lo. Acredito, particularmente, que, para alguns casos selecionados, a operaçāo de Cabrol seja utilizada com baixo risco; entretanto, não gostaria de substituí-la definitivamente, pois considero nossos resultados imediatos e tardios excelentes. Finalmente, agradeço à Comissão Organizadora do Congresso, pela oportunidade que nos ofereceu. 